CASE REPORT

\author{
F.Y. Lin \\ E.M. Genden \\ W.L. Lawson \\ P. Som \\ L. Kostakoglu
}

\title{
High Uptake in Schneiderian Papillomas of the Maxillary Sinus on Positron-Emission Tomography Using Fluorodeoxyglucose
}

\begin{abstract}
SUMMARY: Schneiderian papillomas are benign tumors of the nasal cavity and paranasal sinuses often asymptomatic in their early stages. We report a case of a maxillary sinus oncocytic schneiderian papilloma first detected by positron-emission tomography by using fluorodeoxyglucose (FDG). Schneiderian papillomas demonstrate increased FDG uptake, similar to that of other oncocytic tumors, making it important for otolaryngologists and radiologists to realize that high uptake of FDG does not necessarily indicate a malignant lesion.
\end{abstract}

$\mathbf{P}$ ositron-emission tomography (PET) using fluorodeoxyglucose (FDG) combined with CT (FDG-PET/CT) offers the advantage of integrating morphologic and metabolic data at 1 single session. In head and neck cancer, FDG-PET/CT has been shown to be of particular use in tumor staging and restaging. Furthermore, it provides a superior definition of lesion location and the extent of disease, given the complex anatomy and the presence of physiologic FDG uptake in normal structures. ${ }^{1-4}$ However, FDG is not specific to malignant processes and can also accumulate in benign conditions such as infectious processes and benign tumors that exhibit an increased rate of glycolysis. ${ }^{5,6}$ Thus, it is of prime importance to recognize false-positive lesions for an accurate interpretation of FDG-PET/CT at both the initial staging and restaging. In this article, we report a case of a maxillary sinus oncocytic schneiderian papilloma in a patient with squamous cell cancer of the head and neck, whose detection by FDG-PET led to a subsequent histopathologic confirmation. We also review the clinical characteristics of this benign tumor.

\section{Case Report}

A 70-year-old man presented with ear pain, odynophagia, and hoarseness for 6 months. The patient was without any other significant medical history and denied any symptoms of nasal drainage or obstruction, facial pain, or headaches. On physical examination, the patient was found to have enlarged lymph nodes in level II, and on fiber optic nasolaryngoscopy, a large right pyriform sinus mass with fixation of the right vocal cord was observed. The patient was subsequently referred for a PET/CT study for further evaluation of the extent of disease. PET/CT imaging demonstrated a large hypopharyngeal soft-tissue mass as well as 2 large hypermetabolic right-sided level II lymph nodes. Additionally, an unexpected soft-tissue mass was detected within the wall of the right maxillary sinus (Figs 1 and 2), which demonstrated significantly increased FDG uptake (maximum standard uptake value [SUVmax], 28.0), which was highly suggestive of a malignant process, probably of a metastatic nature. A month later, the patient was brought to the operating room for panendoscopy, biopsy of the hypopharyngeal mass, and a right Caldwell-Luc

Received May 25, 2008; accepted after revision June 21.

From the Department of Otolaryngology-Head and Neck Surgery (F.Y.L., E.M.G., W.L.L.), Head and Neck Cancer Center (E.M.G.), and Department of Radiology (P.S., L.K.), Mount Sinai School of Medicine, New York, NY.

Please address correspondence to Fred Y. Lin, MD, Department of Otolaryngology-Head and Neck Surgery, Mount Sinai School of Medicine, One Gustave L. Levy PI, Box 1189, New York, NY 10029; e-mail: fred.lin@mssm.edu

DOl 10.3174/ajnr.A1264

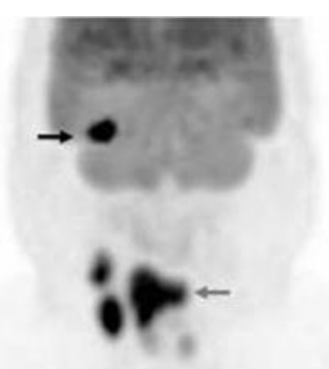

Fig 1. Volumetric FDG-PET image demonstrates increased uptake in the supraglottic/ hypopharyngeal region consistent with the primary tumor (gray arrow). Additionally noted are multiple foci of FDG uptake in right-sided cervical lymph nodes, consistent with metastatic disease. There is an additional focus of intense uptake in the region of the maxilla, suggestive of metastatic disease (black arrow, Fig 2). Faint and symmetric uptake in the midline is consistent with physiologic uptake in the vocal cords.

operation with excisional biopsy of the maxillary sinus mass. Histopathology revealed the hypopharyngeal mass to be a poorly differentiated squamous cell carcinoma. Most interesting, the maxillary sinus mass was histologically proved to be a sinonasal oncocytic schneiderian papilloma. On the basis of these findings, the patient's disease was staged as a T3N2bM0 hypopharyngeal carcinoma. The patient subsequently underwent chemotherapy and hyperfractionated radiation therapy for the hypopharyngeal squamous cell carcinoma followed by a selective neck dissection. He responded well to therapy with no evidence of disease after a follow-up of 8 months, which included repeat PET/CT and diagnostic CT studies. The patient is currently without a recurrence of his maxillary sinus papilloma.

\section{Discussion}

Schneiderian papillomas are benign tumors of the nasal cavity and paranasal sinuses and are often asymptomatic in their early stages. Hyams ${ }^{7}$ described 3 histologic types of sinonasal papillomas: inverted papillomas, fungiform papillomas, and cylindrical cell papillomas. Inverted papillomas, unlike normal papillomas, are derived from the inversion of the neoplastic epithelium into underlying stroma instead of proliferating outward. Fungiform papillomas are exophytic with a thin central core of connective tissue, which is most consistent with the common concept of papillomas. Cylindrical papillomas, which were later described by Barnes and Bedetti ${ }^{8}$ as oncocytic schneiderian papillomas, are characterized by proliferating 

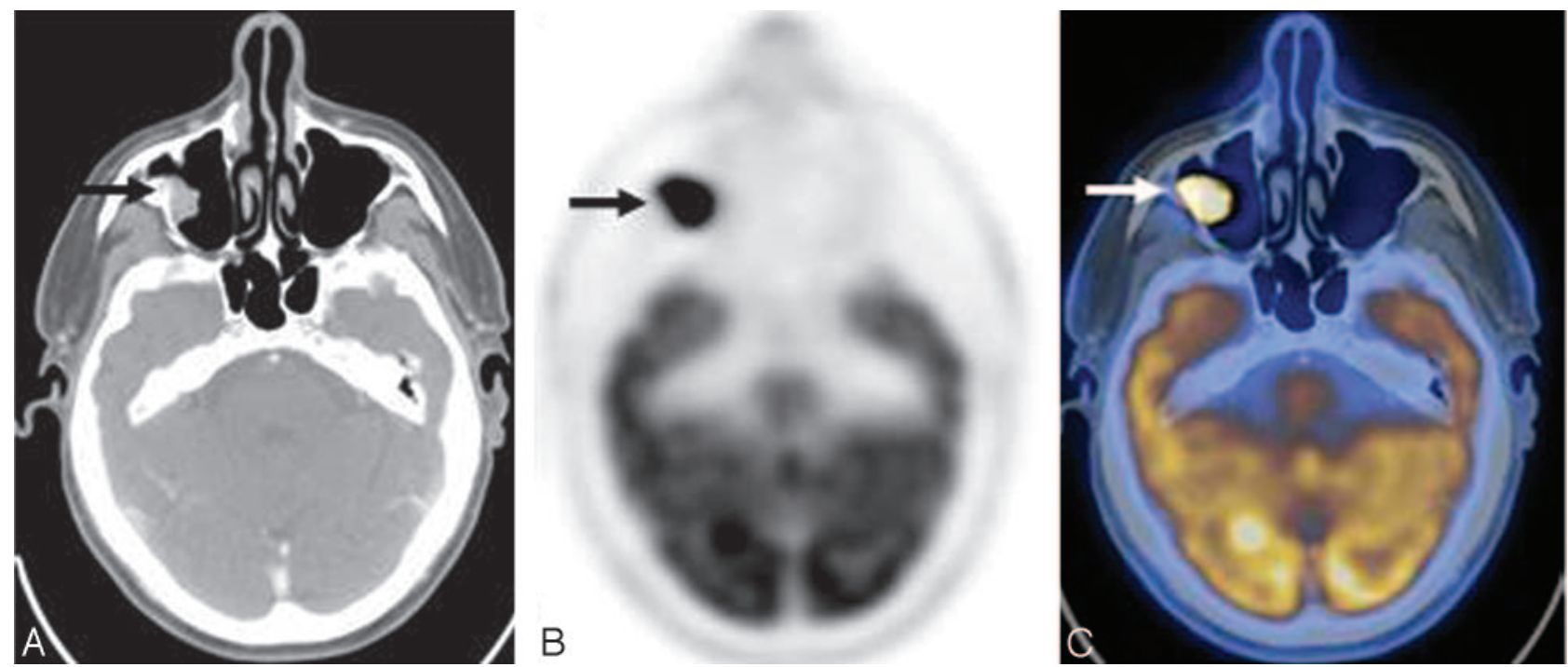

Fig 2. Fusion $(A)$, PET $(B)$, and axial CT $(C)$ images demonstrate a focus of intensely increased FDG uptake (arrow) corresponding to a soft-tissue attenuation projecting off the epithelial surface of the right maxillary sinus, highly suggestive of a malignant process. However, histopathologic examination revealed this mass to be a schneiderian papilloma.

multilayered columnar cells with typical involvement of the maxillary sinus or in conjunction with the lateral wall of the nasal cavity or ethmoid sinus.

Oncocytic schneiderian papillomas are the rarest of the 3 variants, affecting males and females equally, with a propensity for local recurrence (33\%-40\%) and malignant transformation $(4 \%-17 \%))^{7-9}$ Patients presenting with the disease typically offer nonspecific complaints with the most common being nasal obstruction, rhinorrhea, headache and facial pain or hyposmia and, rarely, tinnitus, sensorineural hearing loss, and diplopia. ${ }^{10}$ Symptoms often last between 0 and 72 months, with an average of 24 months, though Eggers et $\mathrm{al}^{10}$ showed that many patients are often asymptomatic at diagnosis.

Although there are no specific radiologic findings of schneiderian papillomas, typical findings on plain radiographs or CT are a unilateral opacification in the maxillary sinus or a mass in the nasal fossa, with occasional thinning or destruction of the bony walls. ${ }^{10} \mathrm{CT}$ is the current technique of choice; however, due to its poor soft-tissue contrast, tumor cannot be differentiated from retained secretions or inflammation. ${ }^{10}$ FDG-PET, on the other hand, can image areas of increased glucose metabolism that appear to be linked with malignancy, though it is not tumor-specific, with uptake being involved in both tumor cells and nonneoplastic cells such as fibroblasts and neutrophils. ${ }^{11}$

Ninomiya et $\mathrm{al}^{11}$ studied 22 patients with suggestion of malignant tumors in the nasal cavity and paranasal sinuses, with results showing malignant tumors having a higher uptake of FDG than those of benign origin. FDG-PET depicted squamous cell carcinoma with high SUV significantly greater than that of normal tissue and benign lesions. However, oncocytic tumors such as Warthin tumors, oncocytomas, and oncocytic papillomas have been observed with high SUVs, despite being benign tumors. ${ }^{12-14}$ The etiology of increased FDG uptake is poorly understood, though it is hypothesized that oncocytic tumors possess a high number of mitochondria causing in- creased metabolism and an increased uptake of metabolites such as FDG. ${ }^{15}$

\section{Conclusions}

FDG-PET has been increasingly used in routine clinical applications for head and neck carcinomas at both initial presentation and follow-up for detection of possible recurrence. It offers a technique of imaging that uses both metabolic and morphologic data, allowing better characterization and localization of tumors. However, increased FDG uptake is not solely limited to malignant lesions. As we report here, schneiderian papillomas, which are rare benign tumors of the nasal cavity and paranasal sinuses, demonstrate significantly increased FDG uptake, similar to that of other oncocytic tumors such as Warthin tumors and oncocytomas. It is important that otolaryngologists and radiologists alike be aware that high uptake of FDG does not necessarily confer a malignant etiology. Thus, these tumors should be included in the differential diagnosis to avoid false readings at both staging and restaging for better patient management.

\section{References}

1. Branstetter BF, Blodgett TM, Zimmer LA, et al. Head and neck malignancy: is PET/CT more accurate than PET or CT alone? Radiology 2005;235:580-86

2. Jeong HS, Baek CH, Son YI, et al. Use of integrated 18F-FDG PET/CT to improve the accuracy of initial cervical nodal evaluation in patients with head and neck squamous cell carcinoma. Head Neck 2007;29:203-10

3. Syed R, Bomanji JB, Nagabhushan N, et al. Impact of combined (18)F-FDG PET/CT in head and neck tumours. Br J Cancer 2005;92:1046-50

4. Hain SF. Positron emission tomography in cancer of the head and neck. $\mathrm{Br} \mathrm{J}$ Oral Maxillofac Surg 2005;43:1-6

5. Fukui MB, Blodgett TM, Snyderman CH, et al. Combined PET-CT in the head and neck. Part 2. Diagnostic uses and pitfalls of oncologic imaging. Radiographics 2005;25:913-30

6. Blodgett TM, Fukui MB, Snyderman CH, et al. Combined PET-CT in the head and neck. Part 1. Physiologic, altered physiologic, and artifactual FDG uptake. Radiographics 2005; 25:897-912

7. Hyams VJ. Papillomas of the nasal cavity and paranasal sinuses: a clinicopathological study of 315 cases. Ann Otol Rhinol Laryngol 1971;80:192-206

8. Barnes L, Bedetti C. Oncocytic schneiderian papilloma: a reappraisal of cylindrical cell papilloma of the sinonasal tract. Hum Pathol 1984;15:344-51 
9. Kapedia SB, Barnes L, Pelzman K, et al. Carcinoma ex oncocytic schneiderian (cylindrical cell) papilloma. Am J Otolaryngol 1993;332-38

10. Eggers G, Muhling J, Hassfeld S. Inverted papillomas of the paranasal sinuses. J Craniomaxillofac Surg 2007;35:21-29. Epub 2007 Jan 30

11. Ninomiya H, Oriuchi N, Kahn N, et al. Diagnosis of tumor in the nasal cavity and paranasal sinuses with $[11 \mathrm{C}]$ choline PET: compared with 2-[18F] flouro2-deoxy-D-glucose (FDG) PET. Ann Nucl Med 2004;19:29-34

12. Noji T, Kondo S, Hirano S, et al. Intraductal oncocytic papillary neoplasm of the pancreas shows positivity on FDG-PET. Int $J$ Gastroinest Cancer 2002;32:43-46

13. Scott GC, Meier DA, Dickinson CZ. Cervical lymph node metastasis of thyroid papillary carcinoma imaged with fluorine-18-FDG, technetium-99m-pertechnetate and iodine-141-sodium iodide. J Nucl Med 1995;36:1843-45

14. San Pedro EC, Lorberboym M, Maschac J, et al. Imaging of multiple bilateral parotid gland oncocytomas. Clin Nucl Med 1995;20:515-18 